\title{
Prescribing psychotropic drugs for the elderly
}

\section{Alistair Burns \& Robert Baldwin}

The pharmacological treatment of the elderly differs from that of younger patients, mainly because of altered pharmacokinetics and the higher proportion of patients with organic disorders. We deal here with dementia, delirium, affective disorder, late-life schizophrenia, sleep disturbance, and rapid tranquillisation.

\section{Dementia}

The commonest forms of dementia are Alzheimer's disease and vascular dementia; Lewy body dementia is also common. Pharmacological therapy (see Whalley (1989) for a comprehensive review) can be directed at two areas:

(1) the cognitive deficit

(2) non-cognitive features - psychiatric symptoms and behavioural disturbances.

\section{Cognitive deficits}

Alzheimer's disease

Treatments aimed at reversing the cognitive deficit have been disappointing. In Alzheimer's disease, most treatments which have shown some efficacy are based on the cholinergic hypothesis, with the common goal of increasing available acetylcholine. Three approaches have been described:

(1) loading with acetylcholine precursors

(2) cholinesterase inhibitors, which inhibit the enzyme that degrades acetylcholine

(3) direct stimulation of the receptor.

Precursors such as lethicin showed early promise, but because of inconsistent results from drug trials and comparative lack of efficacy, these have not become popular. Anticholinesterases have been most studied and have given the most promising results. No anticholinesterase is currently available, although tetrahydroaminoacridine has been most
Box 1. Key learning points

In Alzheimer's disease, there is no effective treatment for the cognitive deficit.

Non-cognitive features in dementia are amenable to treatment with neuroleptics and non-neuroleptic drugs.

In vascular dementia, risk factor reduction and aspirin improve cognitive function.

Patients with Lewy body dementia are particularly sensitive to neuroleptics.

Remember inter-individual variation in drug metabolism is much greater in elderly than in younger patients.

All antidepressant and antipsychotic agents are equally effective, so prescribing decisions are largely based on side-effect profiles.

The principles of prescribing to older patients are not different but are complicated by issues of comorbidity, poorer compliance and more risk of side -effects.

Suicide risk is not dealt with simply by prescribing a 'safer' drug.

investigated and has appeared in the USA under the trade name Cognex. Trials have shown the drug to improve cognition by approximately the same magnitude as the decline which would have been expected over the same period. Side-effects include gastrointestinal disturbances and liver damage.

Several other drugs have been tried in Alzheimer's disease, none of which can be recommended for routine use. Hydergine is regularly prescribed in continental Europe and has been shown, in doubleblind trials, to be effective at reducing anxiety, depression, 'confusion' and 'impaired social care'. While these effects can be of practical benefit to patients and carers, there is little evidence that Hydergine fundamentally alters cognitive function.

Recent negative results have been found in trials with thiamine and piramiracetam but improvements in cognition have been described with 
desferrioxamine, indomethacin and $\mathrm{N}$-acetylcarnitine (for review, see Burns, 1992).

\section{Vascular dementia}

There is evidence that treating risk factors for stroke may improve cognitive impairment. Reducing systolic blood pressure to between 135 and 150 $\mathrm{mmHg}$ improved cognitive function, but further reduction was associated with a deterioration (Meyer et al, 1986). Cessation of smoking in normotensive patients with multi-infarct dementia was also beneficial.

There is evidence that $\mathbf{3 2 5} \mathbf{~ m g}$ aspirin per day improves cognitive function and cerebral perfusion (Meyer et al, 1989). Low-dose aspirin (75 mg per day) reduces the risk of stroke and death in patients with pre-existing vascular disease (SALT, 1991).

\section{Lewy body dementia}

Clinical criteria for Lewy body dementia include episodic confusional states, prominent hallucinations, cognitive loss in the absence of aetiological factors for delirium, and clinical deterioration over time. McKeith et al (1992) reported high mortality in this group of patients when neuroleptics in traditional doses were prescribed. It seems that sufferers have a profound cholinergic loss, and patients with Alzheimer's disease who respond to tetrahydroaminoacridine appear to have Lewy bodies at post-mortem (Levy, 1993). In view of the fluctuation in cognitive state in patients with Lewy body dementia, there are difficulties in attributing improvement to specific medication. The Parkinsonian syndrome in Lewy body disease may respond to L-deprenyl and behavioural disturbances can be controlled with chlormethiazole and benzodiazepines.

\section{Non-cognitive features}

Non-cognitive features of dementia comprise psychiatric symptoms and behavioural disturbances (for review see Burns, 1993). It is important to define accurately those non-cognitive features at which specific treatments are directed.

\section{Neuroleptics}

Neuroleptics have traditionally been prescribed for agitation and aggression, and are moderately effective. Schneider et al (1990) reported a meta-analysis of controlled trials to assess the efficacy of neuroleptics in dementia, and suggested that $18 \%$ of patients with agitated dementia would benefit from these drugs. Individual studies showed no significant change, but overall there was a small effect in favour of neuroleptics.

\section{Box 2. Good practice points}

'Start low, go slow' when prescribing to elderly patients.

Exclude Lewy body dementia, as far as possible, before prescribing neuroleptics to a patient with dementia.

Examine a patient with dementia to exclude vascular disease - treatment of risk factors and/or aspirin may help.

Gain experience with prescribing at least one of the tricyclics and one of the newer agents; become familiar with their dose ranges and side-effect profiles.

Take a good drug and alcohol history polypharmacy accounts for much iatrogenic morbidity.

A therapeutic antidepressant trial in older patients should last at least 6-8 weeks.

A reasonable period for continuation treatment is 12 months.

Lithium dose ranges are lower in the elderly - generally $0.4-0.6 \mathrm{mmol} / 1$.

The efficacy of these drugs is further supported by evidence of a deterioration in behaviour when they are stopped. There is no difference between thioridazine and haloperidol. Lower doses are required to treat the behavioural effects of dementia compared with doses for psychotic illness. There is also evidence that very low doses (e.g. $5 \mathrm{mg}$ thioridazine or $0.125 \mathrm{mg}$ haloperidol) can be effective.

\section{Choice of agent}

This depends on preference and experience. Thioridazine is often prescribed, but it has marked anticholinergic effects; haloperidol has a particular tendency to cause extrapyramidal signs and symptoms; chlorpromazine causes hypotension; and promazine is often regarded as not being of adequate strength (see Table 2). There is little experience with the newer agent clozapine, although there are good theoretical reasons why this might be of benefit in patients with Lewy body disease.

Depot neuroleptics have been tried with some success, and have the advantage of compliance. Sulpiride has been helpful where there is a need to avoid Parkinsonian side-effects.

\section{Non-neuroleptic medication}

Agents other than neuroleptics have been used for control of behavioural symptoms in dementia (Schneider \& Sobin, 1994). These include antidepressants (used not only for affective symptoms), 
Box 3. Controversial issues

Tetrahydroaminoacridine may be helpful in alleviating the cognitive deficit in Alzheimer's disease - a careful diagnostic assessment and follow-up is necessary.

The newer antidepressants are regarded by some but by no means all old age psychiatrists as drugs of first choice when prescribing to elderly depressives.

There is a trend to life-long maintenance therapy in major depression of later life, but not all agree with this.

anticonvulsants, beta-blockers, benzodiazepines, and psychostimulants.

Antidepressants have consistently been shown to be effective in alleviating affective symptoms. Monoamine oxidase inhibitors (types A and B) improve memory and concentration, possibly as a consequence of alleviating depressive symptoms. Selective serotonin reuptake inhibitors have also been used to good effect in reducing aggression and irritability. It has been suggested that they are particularly effective in vascular dementia. Lithium has been shown in a few reports to control agitation, often dramatically, but the side-effects and the proven benefit of other drugs make it an unlikely choice. Beta-blockers have been used with younger patients to control aggression (usually after brain damage), and carbamazepine has been reported to control aggression in a study of about 50 patients; however, the one placebo-controlled study found no improvement. Benzodiazepines may cause confusional states in the elderly and so their use is limited, although, of three recent studies, two have shown benzodiazepines may be more efficacious than thioridazine or haloperidol. Psychostimulants such as methylphenidate have been shown to benefit patients in open trials only, and it is likely with the advent of newer drugs that these older agents will no longer be used. Buspirone has been reported to be beneficial in two out of three cases of dementia. Attention to the serotonergic deficit in Alzheimer's disease has encouraged the use of trazodone (with or without tryptophan) with some improvement in aggression, but this is not limited to dementia.

\section{Delirium}

Delirium is caused by physical disease and, as such, treatment should be primarily aimed at treating this.
Appropriate nursing and medical care is the priority, and where practicable a quiet, well lit environment should be made available. Where behavioural disturbance is prominent, psychotropic medication is indicated. Some conditions, such as delirium tremens or other withdrawal effects, require drug detoxification regimens along conventional lines, but with corresponding dose modification (generally half, or less). Emergent behavioural disorder during delirium should be managed as described under 'Rapid tranquillisation' (see below). Haloperidol and thioridazine are the drugs of choice (chlorpromazine may produce dangerous hypotension).

\section{Depressive illness}

\section{Acute treatment}

Here, depressive illness is used synonymously with major depressive disorder (APA, 1987) and depressive episode (WHO, 1992). However, as with younger patients, those falling somewhat short of criteria for major depressive disorder should be considered for treatment (NIH, 1992).

The following general points are relevant to prescribing:

(1) the adult recovery rate with an antidepressant drug is similar at any age, at around $60-70 \%$

(2) there is no evidence that any drug or class of drugs is superior in terms of response rate to another (Katona, 1993)

(3) predicting the optimal dose of tricyclic antidepressants can be more problematic with elderly patients because of the greater variation between them in drug metabolism and distribution.

The adage 'start low, go slow' is particularly apt for this area of pharmacotherapy, but equally nonreponders may require dosages comparable to those used with much younger adults; some of the newer agents require no dose adjustment for elderly patients. Typical drug starting dosages and therapeutic ranges are shown in Table 1.

Antidepressants can be classified as:

(1) tricyclics, subdivided into the traditional agents (amitriptyline, imipramine, dothiepin, doxepin, clomipramine, nortriptyline) and the newer tricyclic, lofepramine

(2) atypical antidepressants (e.g. trazodone and mianserin) 
Table 1. Main antidepressant inrently used in geriatric practice

\begin{tabular}{|c|c|c|c|c|c|}
\hline Agent & $\begin{array}{l}\text { Anticholinergic } \\
\text { effects }^{1}\end{array}$ & $\begin{array}{l}\text { Orthostatic } \\
\text { effects }{ }^{1}\end{array}$ & Sedation $^{1}$ & $\begin{array}{c}\text { Initial } \\
\text { dose: } \mathbf{m g}\end{array}$ & $\begin{array}{l}\text { Typical dose: } \\
\text { mg/day }\end{array}$ \\
\hline Amitriptyline & 5 & 5 & 5 & 25 & $50-100$ \\
\hline Imipramine & 4 & 5 & 2 & $25-50$ & 50-100 \\
\hline Dothiepin & 4 & 4 & 3 & $25-50$ & $50-100$ \\
\hline Mianserin & $0 / 1$ & $0 / 1$ & 4 & $20-30$ & $30-90$ \\
\hline Lofepramine & $1 / 2$ & 1 & 1 & 70 & $140-210$ \\
\hline Trazodone & 0 & 1 & 3 & $50-100$ & $100-300$ \\
\hline Fluvoxamine & $0 / 1$ & 0 & 0 & 100 & $100-300$ \\
\hline Sertraline & $0 / 1$ & 0 & 0 & 50 & $100-150$ \\
\hline Fluoxetine & $0 / 1$ & 0 & 0 & 20 & 20 \\
\hline Paroxetine & $0 / 1$ & 0 & 0 & 20 & $20-30$ \\
\hline Moclobemide & 1 & 1 & $1 / 2$ & 300 & $300-600$ \\
\hline
\end{tabular}

1. Numbered 0 to 5 by increasing intensity of effect.

(3) the selective serotonin reuptake inhibitors (SSRIs) (fluoxetine, fluvoxamine, paroxetine and sertraline)

(4) monoamine oxidase inhibitors (MAOIs), which again can be subdivided into the traditional agents (phenelzine, tranylcypromine) and the newer, reversible inhibitors of monoamine oxidase A enzyme (RIMA agents), of which moclobemide is the first to be licensed in the UK.

\section{Choice of drug}

\section{Efficacy}

Some concern has been expressed that SSRIs may be less effective in severe depression, but there are as yet no data on elderly patients. There is little doubt that the newer agents (SSRIs, mianserin, trazodone, lofepramine) are safer than the traditional tricyclics in overdosage. However, argument that the latter should be abandoned in favour of newer, safer drugs may be misplaced if it leads to a complacent approach to managing suicide risk, which is high in the elderly.

\section{Contraindications}

Contraindications for tricyclics include a history of acute (but not necessarily chronic) glaucoma; a myocardial infarct within the preceding three months; a tachy- or bradyarrhythmia; poorly controlled (but not necessarily stable) heart failure; or a clinically relevant cardiac conduction disorder (e.g. bi- or trifascicular block); and, in men, prostatism. Severe renal or hepatic insufficiency are contraindications to the use of SSRIs.

\section{Side-effects}

There are important differences in side-effects (Table 1). For the traditional tricyclics, anticholinergic effects are prominent and frequently troublesome, but are less marked with lofepramine. Postural hypotension is arguably the most dangerous sideeffect of the tricyclics. They commonly cause sedation, which is also an effect of trazodone. Tricyclics may provoke delirium, especially in the setting of acute physical illness or dementia; amitriptyline is the worst offender.

With relatively minor variations, all the SSRIs may cause nausea (5-15\%), diarrhoea (10\%), insomnia (5-15\%), agitation and/or anxiety (2-15\%), headache, and weight loss. Many of these are transient and early phenomena. Also, dosages of SSRIs were originally set too high, possibly exaggerating this profile. However, studies of elderly patients have not generally confirmed a lower rate of overall sideeffects of SSRIs when compared with older tricyclics, just a different pattern (Katona, 1993). Sedation is least likely with fluoxetine and more likely with paroxetine, while fluoxetine may lead to anxiety and agitation. Fluvoxamine causes nausea but has a lower incidence of insomnia. Paroxetine is the only SSRI with a specific data-sheet indication for the treatment of anxiety in association with depression.

Although rare, there have been reports of SSRIs either leading to or exacerbating Parkinsonism and akathisia.

The RIMA moclobemide appears to be of equal efficacy to tricyclics and SSRIs in late-life depression, has a low risk of the 'cheese effect', a short duration of action and half-life, and is safe and seems well 
tolerated. Adverse effects include agitation, anxiety, and insomnia.

\section{Interactions}

If changing to a MAOI from fluoxetine, five weeks must elapse; equivalent periods are two weeks for paroxetine, and a week for sertraline. Mixing an SSRI with a MAOI may cause the serotonergic syndrome, characterised by excitement, confusion, rigidity, tremor, hyperthermia, tachycardia, hypotension, and convulsions.

All antidepressants may antagonise anticonvulsant medication. Concomitant antipsychotics raise plasma levels of tricyclic drugs, and the plasma concentration of haloperidol may be increased by fluoxetine. There is an increased risk of lithium toxicity if combined with SSRIs. Two of the latter, fluvoxamine and paroxetine, may enhance the effects of warfarin. Plasma concentrations of lipophilic beta-blockers may be increased by fluvoxamine. Imipramine and possibly other tricyclics may lead to increased levels of diltiazem and verapamil.

Anxiolytics and hypnotics may enhance the sedative effects of tricyclics. Cimetidine may raise plasma levels of moclobemide and the tricyclics.

Although the risk of a hypertensive crisis is low, drugs with sympathomimetic effect and opiatebased drugs should be avoided with moclobemide.

\section{Compliance}

Lack of compliance correlates with the number of other drugs prescribed, and polypharmacy is frequent among older depressed patients. Memory is less efficient in major depressive disorder and may further impair compliance. The once-daily dosaging regimen of most of the SSRIs thus gives them an advantage over tricyclics.

\section{Costs}

The newer agents are generally more expensive. While it has been argued that cost-benefit analysis favours the newer agents because of (alleged) better compliance, there is no evidence regarding older depressed patients.

\section{Comorbidity}

There is evidence that tricyclics are poorly tolerated by elderly medically ill patients (Koenig et al, 1989), but the SSRIs rather better so (Evans, 1993).

There is little literature about how best to treat depression comorbid with dementia. In a doubleblind placebo-controlled trial, Reiffler et al (1989) reported significant improvement in mood in an elderly group of patients with Alzheimer's disease and depression using imipramine. The mean dose was $80 \mathrm{mg}$ daily, but it was noted that cognitive function remained stable only within a fairly narrow dose range.

It is wise then to begin with very small doses of tricyclics (10-20 mg imipramine equivalent) and proceed with small increments. Other agents have been inadequately assessed in dementia. One open study of 10 patients (Postma \& Vranesic, 1985) reported improvement in depression comorbid with dementia using 150-225 mg moclobemide.

\section{Treatment resistance}

Arbitrarily, this is defined as failure to respond after eight weeks of adequate therapy with a single agent. There has been interest in augmentation regimens, but little data pertinent to older depressives. Finch \& Katona (1989) reported improvement and good tolerance in six of nine patients resistant to either first-line treatment or electroconvulsive therapy, or both. Van Marwijk et al (1990) reported a similar improvement rate in a larger series, but encountered more side-effects, especially of lithium toxicity. Seth et al (1992), in an open study of eight refractory, mainly elderly patients, reported improvement in all when given nortripyline plus an SSRI. However, the combination may cause toxic plasma levels of the tricyclic.

\section{Continuation therapy}

Response to treatment is often later than in younger patients (Flint, 1992; NIH, 1992). A minimum trial is six weeks, although a significant minority show response up to eight or nine weeks (NIH, 1992). The standard six months of continuation therapy after resolution of depressive symptoms is probably too short for elderly patients (Flint, 1992); a year is more realistic. The main risk period for recurrence and relapse is the first two years (Flint, 1992).

\section{Prophylaxis}

Some clinicians argue for long-term treatment for all patients who have had major depressive disorder in later life. Few elderly patients remain symptom free during extended periods of follow-up (Baldwin \& Jolley, 1986) and for elderly people one further episode of depressive illness is time lost from an already short life expectancy.

Clinicians are thus faced with the choice of either maintaining patients on antidepressants indefinitely, or attempting to wean them off after about a year. As a guide, those patients who do best are characterised by having good health and an uncomplicated recovery from depression (Baldwin \& Jolley, 1986). Both lithium (Abou-Saleh \& Coppen, 
1983) and dothiepin (OADIG, 1993) are reported to reduce relapse rates significantly in the medium term. For antidepressants the prophylactic dose should be as close as possible to the therapeutic one (Reynolds et al, 1993). However, weight gain associated with long-term tricyclic usage may be problematic; more work is needed on the role of the newer agents in prophylaxis.

A meta-analysis of the efficacy and acceptability of SSRIs (Song et al, 1993) concluded that their routine use as first-line agents in depressive illness was unwarranted. Tricyclic antidepressants can be recommended as well tried, effective and cheap treatments for depressions in old age, provided the patient has no contraindications and tolerates the sideeffects. In practice, the newer agents constitute an important advance in the treatment of those elderly patients, not inconsiderable in number, who do not fulfil these conditions, especially the physically frail.

\section{Mania}

\section{Acute treatment}

Neuroleptic drugs are the mainstay of treatment in this phase. Haloperidol, which has an extremely wide dose range, is popular. Drugs with lower potency may be preferred, such as thioridazine or promazine, to reduce the risk of extrapyramidal problems. Practical problems include falls caused by a mixture of Parkinsonism and sedation in an overactive patient, and anticholinergic delirium, caused by co-prescribing neuroleptics and anticholinergic agents (benzhexol, orphenadrine, benztropine) - an eruption of manic symptoms may then be erroneously diagnosed. Lithium is a further option for the less disturbed patient, but serum levels should be lower than for younger patients on average $0.4-0.6 \mathrm{mmol} / 1$ (Shulman et al, 1992). There is a little evidence that valproate or carbamazepine may be effective, but much further work is required, including research to produce definitive guidelines for lithium treatment in older people (Shulman et al, 1992).

\section{Prophylaxis}

Lithium may be used in prophylaxis. As with acute treatment, dosages will generally be half those for younger adults. Lithium toxicity is a risk and is associated as much with poor monitoring as with organic (not infrequently cerebral) pathology. Regular checks of renal and thyroid function and concurrent medication, particularly the inadvertent prescription of a diuretic, are essential.

\section{Late-life schizophrenia (paraphrenia)}

The term 'paraphrenia' may or may not survive as a nosological entity, but clinicians working with the elderly recognise a specific type of psychotic disorder arising in later life.

\section{Acute treatment}

The principles of treating schizophrenia are similar at any age. Cerebral ageing results in reduced levels of dopamine and tyrolase hydroxylase, as well as lower counts of dopamine-rich neurons in the midbrain (Morgan et al, 1987). The overall effect is an increased susceptibility to neuroleptic-induced extrapyramidal disorder in elderly patients, with the exception of acute dystonic reactions, which are rare.

Antipsychotic drugs can be classified into:

(1) phenothiazines, subdivided as aminoalkyl compounds (e.g. chlorpromazine), piperazines (e.g. trifluoperazine) and piperidines (e.g. thioridazine)

(2) thioxanthenes (e.g. flupenthixol)

(3) butyrophenones (e.g. haloperidol and pimozide, a butyrophenone derivative)

(4) dibenzapines (clozapine).

Other, newer agents are sulpiride (a substituted $\mathrm{O}$ anisomide) and risperidone (a benzisoxazole). Remoxipride has been withdrawn. The first three groups have similar pharmacokinetics and undergo hepatic metabolism and renal excretion of metabolites. Their elimination half-lives are in the order of $10-30$ hours, although that of pimozide is much longer and those of clozapine and sulpiride somewhat shorter (5-15 hours). The risk of tardive dyskinesia (TD) in the elderly may be less with clozapine. Clinical experience with risperidone in the elderly is limited. Typical starting doses and dose ranges are shown in Table 2.

\section{Choice of drug}

Efficacy

All current antipsychotics are effective in late-onset schizophrenia.

\section{Contraindications}

Caution is necessary (in particular with pimozide) where there is a history of myocardial disease, hepatic disorder, prostatism and Parkinson's disease, 
Table 2. Dosages and effects of antipsychotic drugs

\begin{tabular}{|c|c|c|c|c|c|c|}
\hline Drug & $\begin{array}{c}\text { Anti- } \\
\text { dopaminergic }\end{array}$ & $\begin{array}{l}\text { Anti- } \\
\text { muscarinic }\end{array}$ & $\begin{array}{c}\text { Anti- } \\
\text { histaminic }\end{array}$ & $\begin{array}{l}\text { Anti- } \alpha \text { - } \\
\text { adrenergic }\end{array}$ & $\begin{array}{c}\text { Suggested } \\
\text { starting doses: } \\
\text { mg/day }\end{array}$ & $\begin{array}{l}\text { Suggested } \\
\text { therapeutic } \\
\text { range: } \mathrm{mg} / \text { day }\end{array}$ \\
\hline Chlorpromazine & ++ & + & ++ & +++ & 25 & $25-200$ \\
\hline Thioridazine & + & ++ & ++ & ++ & $25-50$ & $75-200$ \\
\hline Perphenazine & ++ & + & ++ & + & 4 & 4-12 \\
\hline Trifluoperazine & +++ & + & + & + & 2 & $5-10$ \\
\hline Haloperidol & +++ & \pm & \pm & + & 3 & $3-10$ \\
\hline Pimozide & ++ & \pm & + & + & 2 & $2-8$ \\
\hline Sulpiride & + & \pm & 0 & 0 & 100 & $200-400$ \\
\hline Clozapine & + & ++ & ++ & + & 25 & $100-200$ \\
\hline Promazine & + & + & +++ & + & $25-50$ & 300 \\
\hline
\end{tabular}

+++ , marked; ++ , moderate; + , mild \pm minimal; 0 , none.

but apart from agranulocytosis most contraindications are relative.

\section{Side-effects}

In practice, choosing an agent will depend on the profile of anticipated side-effects. These are best understood by reference to individual neurotransmitter systems (Table 2). Antidopaminergic effects cause Parkinsonism and akathisia. The former is a particular hazard for elderly patients. Lowering the dose, if practicable, is the first move; otherwise changing to a lower-potency neuroleptic may help. Routine prescribing of anticholinergic agents to suppress such symptoms is not recommended, and may increase the risk of TD in older patients (WHO, 1990). Age is a major risk factor for TD, so that drug treatment should be reviewed regularly. 'Drug holidays' may make the problem worse. Treatment of TD is difficult but sulpiride may benefit some patients (Schwartz et al, 1990).

Anticholinergic effects cause problems similar to those outlined for tricyclic antidepressants. Antihistaminic effects may lead to oversedation, especially when combined with other centrally active drugs, including antidepressants and hypnotics. Weight gain may occur with long-term use. Lastly, antiadrenergic effects may cause dangerous postural hypotension. Chlorpromazine is the worst culprit; it is not a drug of first choice for the frail elderly.

In addition, there are idiosyncratic reactions, of which the most important are:

(1) the neuroleptic malignant syndrome

(2) adverse liver effects (which are not confined to chlorpromazine)

(3) agranulocytosis.
Long-term use is associated with corneal particulate material, which could carelessly be attributed to cataracts in the elderly.

\section{Interactions}

Altered plasma levels of antipsychotic may occur with: lithium, phenobarbitol, carbamazepine, phenyldantoin, cimetidine, propranolol, and antidepressant drugs.

\section{Costs}

Many clinicians have locally produced protocols for the use of newer agents, such as risperidone and clozapine, because of their expense.

\section{Treatment resistance}

Clozapine is reserved for refractory schizophrenia and is not contraindicated in older patients, although dosages are typically half those for younger adults, and hypotension can be problematic. Surprisingly, age has not been identified as a risk factor for agranulocytosis (Ball, 1992).

\section{Maintainance therapy}

Post's seminal work (1966) suggested that only a third of paraphrenic patients followed over 14-21 months remained free of symptoms. Regrettably, the advent of newer antipsychotics and specialised services has not convincingly altered this prognosis (Howard \& Levy, 1992).

Howard \& Levy's study of 64 paraphrenic patients (1992) suggests that compliance, and therefore outcome, may be improved by the use of depot neuroleptics and deployment of community psychiatric nurses. All the current depot neuroleptics have been used in elderly patients, and there is little to 
Table 3. Drugs used to treat sleep disorders

\begin{tabular}{|c|c|c|c|c|}
\hline Proprietary name & Trade name & Starting dose & Maximum dose & Side-effects \\
\hline Chloral hydrate & Welldorm & 1-2 tablets nocte & 5 tablets & Gastric irritation \\
\hline Triclofos sodium & Triclofos & $1-2 \mathrm{~g}$ nocte & $2 \mathrm{~g}$ & $\begin{array}{l}\text { Gastric irritation } \\
\text { (but less than with chloral hydrate) }\end{array}$ \\
\hline Chlormethiazole & Heminevrin & 1-2 capsules nocte & 3 capsules & Nasal congestion \\
\hline Zopiclone & Zimovane & $1 / 2$ tablet nocte & 2 tablets & New drug, caution in elderly \\
\hline Temazepam & Temazepam & 5-15 mg nocte & $30 \mathrm{mg}$ & As with other benzodiazepines \\
\hline
\end{tabular}

suggest that one is superior to another, or that any is less likely to precipitate Parkinsonism. Doses will generally be half those for younger adults. Parkinsonism may occur suddenly after weeks of treatment, and may take months to wear off, or may expose latent Parkinson's disease. Many clinicians thus avoid the longer-acting depot agents (haloperidol decanoate, pipothiazine palmitate) in elderly patients.

\section{Sleep disorders}

Disturbed sleep in the elderly is a common complaint, and often results in a request for sedative medication. Sleep disturbances are often secondary to a physical disorder (particular culprits being pain, dysuria, and nocturia) or a psychiatric disturbance (such as depression or delirium). Attention to the primary problem often leads to restoration of the normal sleep pattern.

Iatrogenic causes of sleep disturbance include steroids, theophyllines and psychoactive medication as well as alcohol.

Snoring, sleep apnoea and neuromuscular disorders such akathisia may be responsible for the disturbance.

Specific agents to treat sleep disorders, usually advised on a short-term basis, are chloral hydrate and dichloraphenamide. However, the unpleasant taste and smell and gastric irritation with the former and potential drug interaction (with anticonvulsants and coumarin anticoagulants) with the latter limit their usefulness. Chlormethiazole is effective and widely used, the only particular side-effect being nasal stuffiness. Benzodiazepines are still the most commonly used drugs for sleep disturbance, and the shorter acting the drug the better (temazepam being the best). Chlordiazepoxide and diazepam are longer acting and newer agents such zopiclone require more research. Agents are summarised in Table 3.

\section{Rapid tranquillisation}

The elderly, in common with their younger counterparts, can become aggressive and agitated, and require emergency sedation. One has to exercise more caution with sedation, as coexisting physical illness may interact detrimentally with prescribed medication. For example, heavy sedation in a person with a confusional state secondary to cerebral hypoxia could potentially be fatal. Bearing this in mind, the principles of rapid tranquillisation are the same as in younger patients, although doses need to be adjusted accordingly. Intravenous treatment is rarely required, and intramuscular injections usually suffice. Prolonged and sustained aggression is not as often a management problem, and longterm treatment is rarely necessary.

Haloperidol is particularly effective at controlling aggression, and a suggested regimen for management is:

(1) $1 \mathrm{mg}$ orally every hour (1-5 mg intramuscularly in an emergency)

(2) continue with 0.5 to $2.0 \mathrm{mg} 8$ hourly

(3) when settled for 48 hours, reduce dose by $25 \%$, and continue with this until signs of aggression 'break through'

(4) aim to discontinue drug after a maximum of 4 weeks.

\section{References}

Abou-Saleh, M. T. \& Coppen, A. (1983) The prognosis of depression in old age: the case for lithium therapy. British Journal of Psychiatry, 143, 527-528.

American Psychiatric Association (1987) Diagnostic and Statistical Manual of Mental Disorders (3rd edn, revised) (DSM-III-R). Washington, DC: APA.

Baldwin, R. C. \& Jolley, D. J. (1986) The prognosis of depression in old age. British Journal of Psychiatry, 149, 574-583. 
Ball, C. J. (1992) The use of clozapine in older people. International Journal of Geriatric Psychiatry, 7, 689-692.

Burns, A. (1992) Treatment by physical methods, medication. Current Opinion in Psychiatry, 5, 567-570.

- (1993) Treatment of non-cognitive features of dementia. In Treatment and Care in Old Age Psychiatry (eds R. Levy, R. Howard \& A. Burns), pp. 37-48. Petersfield: Wrightson Biomedical.

Evans, M. E. (1993) Depression in elderly physically ill inpatients, a 12 month prospective study. International Journal of Geriatric Psychiatry, 8, 587-592.

Finch, E. J. L. \& Katona, C. L. E. (1989) Lithium augmentation in the treatment of refractory depression in old age. International Journal of Geriatric Psychiatry, 4, 41-46.

Flint, A. J. (1992) The optimum duration of antidepressant treatment in the elderly. International Journal of Geriatric Psychiatry, 7, 617-619.

Howard, R. \& Levy, R. (1992) Which factors affect treatment response in late paraphrenia. International Journal of Geriatric Psychiatry, 7, 667-672.

Jacoby, R. (1991) Manic illness. In Psychiatry in the Elderly (eds R. Jacoby \& C. Oppenheimer), pp. 720-734. Oxford: Oxford University Press.

Katona, C. L. E. (1993) Optimising treatment for the elderly depressive, new antidepressants in the elderly. Journal of Psychopharmacology, 7 (suppl. 1), 131-134.

Koenig, H. G., Goli, V., Shelp, F., et al (1989) Antidepressant use in elderly medical inpatients, lessons from an attempted clinical trial. Journal of General Internal Medicine, 4, 498-505.

Levy, R. (1993) Is there life in the neuro-transmitter approach to the treatment of Alzheimer's disease? In Treatment and Care in Old Age Psychiatry (eds R. Levy, R. Howard \& A. Burns), pp. 39-46. Petersfield: Wrightson Biomedical.

McKeith, I., Perry, R., Fairbairn, A., et al (1992) Neuroleptic sensitivity with senile dementia of the Lewy body type. British Medical Journal, 305, 673-678.

Meyer, J., Judd, B., Tawaklna, T., et al (1986) Improved cognition after control of risk factors for multi-infarct dementia. Journal of the American Medical Association, 265, 2203-2209.

-, Rogers, R., McClintic, K., et al (1989) Randomised clinical trial of daily aspirin therapy and multi-infarct dementia. Journal of the American Geriatric Society, 37, 549-555.

Morgan, D. G., May, P. C. \& Fich, C. E. (1987) Dopamine and serotonin system in human and rodent brain, effects of age and neurodegenerative disease. Journal of the American Geriatric Society, 35, 334-345.

NIH Consensus Development Panel on Depression in Late Life (1992) Diagnosis and treatment of depression in late life. Journal of the American Medical Association, 268, 1018-1024.

OADIG (1993) How long should the elderly take antidepressants? A double-blind placebo-controlled study of continuation/prophylaxis therapy with dothiepin. British Journal of Psychiatry, 162, 175-182.

Post, F. (1966) Persistent Persecutory States of the Elderly. Oxford: Pergamon.

Postma, J. U. \& Vranesic, D. (1985) Moclobemide in the treatment of depression in demented geriatric patients. Acta Therapeutica, $11,1-4$.

Reiffler, B. V., Teri, L., Raskind, M., et al (1989) Double-blind trial of imipramine in Alzheimer's disease patients with and without depression. American Journal of Psychiatry, 146, 45-49.

Reynolds, C. F., Schneider, L. S., Lebowitz, B. D., et al (1993) Treatment of depression in the elderly, guidelines for primary care. In Diagnosis and Treatment of Depression in the Elderly, Proceedings of the NIH Consensus Development Panel Conference (eds L. S. Schneider, C. F. Reynolds, B. Lebowitz \& A. Friedhoff). Washington, DC: APA.

SALT Collaborative Group (1991) Swedish Aspirin Low Dose Trial of $75 \mathrm{mg}$ aspirin as secondary prophylaxis after cerebrovascular ischaemic events. Lancet, 338, 1345-1349.

Schneider, L., Pollock, D. \& Lyness, S. (1990) A meta-analysis of controlled trials of neuroleptic treatment in dementia. Journal of the American Geriatric Society, 38, 553-563

\& Sobin, P. (1994) Treatment for psychiatric symptoms and behavioural disturbances. In Dementia (eds A. Burns \& R. Levy). London: Chapman and Hall.

Schwartz, M., Moquillansky, L., Lanyi, G., et al (1990) Sulpiride in tardive dyskinesia. Journal of Neurology, Neurosurgery and Psychiatry, 53, 800-802.

Seth, R., Jennings, A. L., Bindman, J., et al (1992) Combination treatment with noradrenaline and serotonin reuptake inhibitors in resistant depression. British Journal of Psychiatry, 161, 562-565.

Shulman, K., Tohen, M. \& Satlin, A. (1992) The significance of mania in old age. Reviews in Clinical Gerontology, 2, 39-43.

Song, F., Freemantle, N., Sheldon, T. A., et al (1993) Selective serotonin reuptake inhibitors, a meta-analysis of efficacy and acceptability. British Medical Journal, 306, 683-687.

Van Marwijk, H. W. J., Beeker, F. M., Nolan, W. A., et al (1990) Lithium augmentation in geriatric depression. Journal of Affective Disorders, 20, 217-223.

Whalley, J. L. (1989) Drug treatments of dementia. British Journal of Psychiatry, 155, 595-611.

World Health Organization (1990) Heads of centres collaborating in WHO co-ordinated studies in biological aspects of mental illness. Prophylactic use of anticholinergics in patients on longterm neuroleptic treatment, a consensus statement. British Journal of Psychiatry, 156, 412.

(1992) The ICD-10 Classification of Mental and Behavioural Disorders. Clinical Descriptions and Diagnostic Guidelines. Geneva: WHO.

\section{Multiple choice questions}

1 The following drugs have proven value in treating non-cognitive features of dementia:
a haloperidol
b lithium
c benzodiazepines
d trazodone
e SSRIs

2 In the treatment of depression in the elderly:

a trazodone has a high level of anticholinergic effects

b SSRIs cause diarrhoea in $30 \%$ of patients

c paroxetine is more sedative than fluoxetine

d fluvoxamine is particularly associated with insomnia

e SSRIs are less effective in severe depression

\section{MCQ answers}

1

a $\mathbf{T}$

b $\mathbf{F}$

c $\mathbf{T}$

d $\mathbf{T}$

e $\mathbf{T}$
2

a $\mathbf{F}$

b $\mathbf{F}$

c $\mathbf{T}$

d $\mathbf{F}$

e $\mathbf{F}$ 